\title{
High Resolution Imaging of Compact Radio Sources by Gridding with Regularization
}

\section{S. F. Likhachev}

Astro Space Center of Lebedev's Physical Institute, Moscow, Russia

R. M. Hjellming

National Radio Astronomy Observatory, Socorro, NM 87801, U.S.A.

Abstract. The problem of VLBI image reconstruction is a classical example of an ill-posed problem. A new procedure of gridding with regularization has been developed. This procedure was used in traditional methods (CLEAN, Hybrid) to improve the quality of compact radio source images. A few sources (GRO J1655-40, RY Scuti and Cyg X-1), observed with the VLA and VLBA, were processed with this procedure.

\section{Introduction}

The main goal of any data processing technique is to get the maximum amount of information from the measurements. Since we usually have no previous knowledge of the characteristics of the radio sources we are investigating, we can rely only on the measurements we obtain. Hence, most VLBI image reconstruction procedures have a subjective nature.

The first paper devoted to the application of the regularization methods for image reconstruction problem in radio astronomy was written by Tikhonov (1969). Tikhonov's regularization methods were first implemented for interferometric imaging by Cornwell (1983).

\section{Gridding with Regularization}

Gridding can be implemented by a regularization procedure that is a convolution of a given function (measured visibility function) with a normalized convolution kernel. In that case, the regularization is a local approximation procedure. tion:

The gridding procedure can be described by the the following approxima-

$$
\sum_{i=1}^{N} w_{i} V_{\text {true }}\left(\mathbf{u}_{i}\right) \varphi\left(k \Delta \mathbf{u}-\mathbf{u}_{i}\right)=V_{\text {grid }}(k \Delta \mathbf{u}), \forall k \in[1, N]
$$

where, $V_{\text {true }}\left(\mathbf{u}_{i}\right)$ is the true unknown visibility and $V_{\text {grid }}(k \Delta \mathbf{u})$ is an approximation of $V_{\text {true }}\left(\mathbf{u}_{i}\right)$.

In other words, it is possible to consider equation (1) as a classical example of a convolution equation with an approximately known right-hand part, i.e., an example of ill-posed problem.

The solution of Equation (1) can be obtained by taking Fourier transform of both parts of the expression and then

$$
I_{\text {true }}(k \Delta \mathbf{x})=\frac{I_{g r i d}(k \Delta \mathbf{x})}{\Phi(k \Delta \mathbf{x})} .
$$


Thus, one obtains a corrected value of the brightness at given grid points. But as shown by Tikhonov (1969) the direct solution of the convolution equation can not be implemented for the case of the ill-posed problem because the solution can not exist, and even if it did exist, it would be unstable. To solve the convolution equation one must use methods from the theory of ill-posed problems.

Let us consider Tikhonov's functional

$$
J=\left\|\sum_{i=1}^{N} w_{i} V_{t r u e}\left(\mathbf{u}_{i}\right) \varphi\left(k \Delta \mathbf{u}-\mathbf{u}_{i}\right)-V_{\text {grid }}(k \Delta \mathbf{u})\right\|^{2}+\alpha\left\|V_{\text {true }}\left(\mathbf{u}_{i}\right)\right\|^{2},
$$

where $\alpha$ is a stabilization parameter, or stabilizer, that can be calculated or chosen empirically. An optimum value of the $\alpha$ parameter can be chosen based on the experimental errors. After simple transformations, it is possible to show (Tikhonov 1969, Likhachev 1996) that

$$
I_{\text {true }}(k \Delta \mathbf{x})=\frac{I_{g r i d}(k \Delta \mathbf{x}) \cdot \Phi(k \Delta \mathbf{x})}{\Phi^{2}(k \Delta \mathbf{x})+\alpha \sum_{n=0}^{p} q_{n} \mathbf{x}^{2 n}}=I_{g r i d}(k \Delta \mathbf{x}) W(k \Delta \mathbf{x}) .
$$

Thus, we have shown that minimization of the functional $J$ (cf. Equation 3 ) is equivalent to filtering with a transfer function.

It is also possible to apply the filter $W(k \Delta \mathbf{x})$ (i.e., the regularization operator $R_{\alpha}$ ) to regularize the synthesized beam.

Gridding with regularization can be added to traditional processing methods (CLEAN and Hybrid). We have done this and applied it to radio source data, for GRO J1655-40, RY Scuti and CygX-1, obtained with the VLA and the VLBA. The improvements in imaging for each of these sources are somewhat different, illustrating the fact that this type of image processing is very dependent on the true nature of the sources. For the highly linear, superluminal radio jets of GRO J1655-40 it is the localization of the imaging to a line that allows even the dirty map to provide a reasonably good image of the source (Hjellming \& Rupen 1995). For the torus of ionized emission around the close binary RY Scuti, the improved resolution of gridding with regularization better emphasizes details in the nebulosity (Gehrz 1995). The field near Cyg X-1 contains a very extended source to the east, and another compact source to the south; imaging using regularization for three fields allows one to optimally image the three sources. These experimental results can be found on the Web page: http://www.asc.rssi.ru/slikhach/aslpics.

Acknowledgments. The National Radio Astronomy Observatory is a facility of the NSF, operated under a cooperative agreement by Associated Universities, Inc.

\section{References}

Tikhonov, A. N., et al. 1969. Soviet Astronomy AJ, 13, 374-380.

Cornwell, T. 1983. A६A, 121, 281-285.

Likhachev, S. F. 1996. ASC FIAN preprintemacs \#22, 11p.

Hjellming, R. M., \& Rupen, M.P. 1995. Nature, 375, 464-468.

Gehrz, R. D., et al. 1995. ApJ, 439, 417-430. 